\title{
Aus Sachsen in die Welt - das sächsische Landesdigitalisierungsprogramm
}

Trotz mancher Zerstörungen durch Kriege und Misswirtschaft ist Sachsen bis heute eine der reichsten deutschen Kulturlandschaften. Durch eine frühe Industrialisierung, einen starken unternehmerischen Mittelstand und viele lebendige Wirtschafts- und Kulturzentren besaß das Königreich Sachsen zu Beginn des 20. Jahrhunderts die größte Industriedichte und das höchste Pro-Kopf-Einkommen im Deutschen Reich. In Sachsen befanden sich zum Beispiel die Wiege des deutschen Maschinenbaus, ein Zentrum der Textilindustrie, die deutsche Druckund Verlagshauptstadt sowie die erste internationale Mustermesse (Karlsch 2006). Den auch außerhalb der Metropolen bewahrten historischen Zeugnissen im digitalen Zeitalter Sichtbarkeit und Reichweite zu verschaffen, ist ein wesentliches Ziel des neuen „Landesdigitalisierungsprogramms für Wissenschaft und Kultur des Freistaates Sachsen“. Ohne zentrale Koordination und tatkräftige fachliche Unterstützung würden die meist personal- und finanzschwachen Gedächtniseinrichtungen in Bautzen, Grimma, Görlitz, Meißen, Plauen oder Zwickau die anspruchsvolle Integration ihrer Objekte in eine nachhaltige digitale Informationsinfrastruktur kaum bewältigen können. Für die wissenschaftliche Forschung und schon gar für die Menschen in der jeweiligen Region sind jedoch nicht nur die Schätze der kulturellen Leuchttürme interessant, sondern gerade auch die wertvollen Objekte in der sogenannten Provinz. Mit seinem flächendeckenden koordinierenden Ansatz schließt das sächsische Landesdigitalisierungsprogramm an ältere, ähnlich gerichtete Bemühungen des Freistaates wie zum Beispiel das sächsische Kulturraumgesetz (1993) an (Winterfeld 2006).

\section{Die sächsische Bibliothekslandschaft}

Der kulturellen Vielfalt des Landes entspricht das enge Bibliotheksnetz Sachsens, das schon im 19. und frühen 20. Jahrhundert auch überregionale Impulse setzte. Erinnert sei an die Gründung der ersten deutschen Volksbücherei durch Karl Preusker in Großenhain (1828), den Aufbau der ältesten öffentlichen Blindenbücherei Deutschlands (1894), die Gründung der Deutschen Bücherei als deutscher Nationalbibliothek (1912) sowie die Einrichtung der ersten deutschen bibliothekarischen Ausbildungsstätte (1914). Für die 1920er Jahre ist Martin Bollert zu nennen, der als Direktor der Sächsischen Landesbibliothek die 1556 gegründete 
Institution zu einer der damals modernsten wissenschaftlichen Gebrauchsbibliotheken entwickelte.

Nach NS-Diktatur und Weltkrieg war es zunächst nicht möglich, an die früheren Erfolge anzuknüpfen. Trotz großen persönlichen Engagements und bewundernswerter Kreativität der sächsischen Bibliothekare führten fehlende Investitionen in Bauten und Ausstattung dazu, dass das Leistungsniveau immer weiter hinter den westdeutschen Standard zurückfiel. Räumliche Provisorien blieben bis zur politischen Wende die Regel und auch die in den 1970er Jahren einsetzende Automatisierung der Geschäftsprozesse wurde in der Ära der DDR nur unzureichend vollzogen. Nach 1990 musste daher zunächst die äußere Neugründung der Bibliotheken im Vordergrund stehen. Mit erheblichen Investitionen in den Aufbau von Büchergrundbeständen sowie in Neubau, Sanierung und Einrichtung von Bibliotheksgebäuden wurden die Voraussetzungen geschaffen, um zeitgemäßen Service anbieten und am Wettstreit der deutschen Bibliotheken um die besten Dienstleistungskonzepte und Betriebsergebnisse wieder aussichtsreich teilnehmen zu können. Im Bereich der wissenschaftlichen Bibliotheken gelangen in kurzer Folge die Neubauten der Fachhochschulbibliothek Zwickau (1998), der Sächsischen Landesbibliothek - Staats- und Universitätsbibliothek Dresden (SLUB, 2002), der Fachhochschulbibliothek Zittau/Görlitz (2004/2006), der Fachhochschulbibliothek Dresden (2006) sowie die umfassende Rekonstruktion und Erweiterung des Hauptgebäudes der Universitätsbibliothek Leipzig (2002). Die Bibliotheken der Musikhochschule Dresden (2008) und der Fachhochschule Leipzig (2009) sind inzwischen ebenfalls fertig gestellt, für die Universitätsbibliotheken in Chemnitz und Freiberg laufen die Bau- bzw. die Planungsarbeiten.

Dem Bauboom unmittelbar vorausgegangen war eine notwendige Strukturbereinigung im Hochschul- und Bibliothekssystem. So wurden etwa in Dresden die Hochschule für Verkehrswesen, die Medizinische Akademie und die Pädagogische Hochschule in die Technische Universität integriert, bald darauf die Bibliothek der TU mit der Sächsischen Landesbibliothek zur SLUB zusammengeführt (1996). Heute umfasst der Kreis der wissenschaftlichen Bibliotheken im Freistaat Sachsen neben der SLUB die drei Universitätsbibliotheken Leipzig, Chemnitz und Freiberg, fünf Fachhochschulbibliotheken sowie fünf Kunst- und Musikhochschulbibliotheken. Zugehörig sind ferner zahlreiche kleinere Spezialbibliotheken, wie zum Beispiel Archiv-, Museums- und Kirchenbibliotheken, die Bibliotheken der „Berufsakademie Sachsen“ sowie städtische wissenschaftliche Bibliotheken wie die Oberlausitzische Bibliothek der Wissenschaften in Görlitz und die Ratsschulbibliothek in Zwickau. Während die anderen großen sächsischen Hochschulbibliotheken vornehmlich naturwissenschaftlich-technisch ausgerichtet sind, steht die Bibliothek der 1409 gegründeten Universität Leipzig als eine der ältesten deutschen Universitätsbibliotheken im Zentrum vielfältiger 
geisteswissenschaftlicher Forschungs- und Erschließungsprojekte. Mit ihrem außerordentlich wertvollen Bestand an mittelalterlichen Handschriften, Inkunabeln und Drucken der Frühen Neuzeit, Papyri und Ostraka, Münzen und Autographen ist sie neben der SLUB die zweite herausragende wissenschaftliche Bibliothek des Freistaates Sachsen (Schneider 2009). Für das Landesdigitalisierungsprogramm relevant sind außerdem Öffentliche Bibliotheken mit historischen Kostbarkeiten wie etwa die Stadtbibliotheken in Leipzig und Chemnitz, Bautzen oder Zittau, aber auch Einrichtungen in anderer Trägerschaft, zum Beispiel das Sorbische Institut oder die von der Stadt Glashütte mitgetragene Stiftung „Deutsches Uhrenmuseum Glashütte“.

Nach der aufreibenden Phase der Bibliotheksfusionen, Bestandsbereinigungen und Bauvorbereitungen rückte seit Mitte des letzten Jahrzehnts zunehmend die innere Neugründung der Bibliotheksregion Sachsen in den Mittelpunkt, das heißt die Modernisierung der bibliothekarischen Geschäftsgänge und die vertiefte Zusammenarbeit unter den Bibliotheken. Entsprechend verständigten sich die sächsischen wissenschaftlichen Bibliothekare 2007 in einem umfassenden Struktur- und Entwicklungsplan auf die konsequente Vernetzung und Konzentration ihrer Kräfte und vereinbarten für alle Bereiche der modernen Bibliotheksarbeit konkrete Kooperationsprojekte (Bonte und Linek 2008). Angestrebt wurden die Profilschärfung der sächsischen Bibliothekslandschaft, die Verminderung konzeptioneller Doppelarbeit, raschere Entwicklungserfolge und ein steter Innovationsimpuls gerade für kleinere Häuser. Gemäß ihrer besonderen Ressourcen und der Vorgaben der sächsischen Staatsregierung übernahm die SLUB dabei zentrale Koordinierungs- und Dienstleistungsfunktionen, die in den Novellen des sächsischen Hochschul- (2008) sowie des SLUB-Gesetzes (2014) rechtlich bestätigt wurden. Die ersten Verabredungen des Struktur- und Entwicklungsplans betrafen weithin schon fast üblich gewordene Informationsdienstleistungen, die in der Ära der DDR und in den folgenden Jahren der elementaren Wiederaufbaulasten nicht oder nicht hinreichend angepackt werden konnten. Das insgesamt weitaus umfangreichste, aus Mitteln des Europäischen Fonds für Regionale Entwicklung (EFRE) unterstützte Teilprojekt zielte auf den möglichst kompletten elektronischen Nachweis der sächsischen Bibliotheksbestände. Während in den wissenschaftlichen Bibliotheken der alten Bundesrepublik bereits um 1980 vergleichbare Maßnahmen eingesetzt hatten, wurde die seit Anfang der 1990er Jahre laufende elektronische Verzeichnung der Neuerwerbungen in Sachsen seit 2008 durch eine groß angelegte Erfassung von Altbeständen ergänzt. Fünf Jahre später waren allein aus der Universitätsbibliothek Leipzig und aus der SLUB rund 2,8 Millionen Titel bearbeitet. Ohne EFRE wäre diese Herausforderung in überschaubarer Zeit nicht zu bewältigen gewesen. 


\section{Grundlagen und Ergebnisse der Retro- digitalisierung und Langzeitarchivierung in Sachsen bis 2015}

Nach der Herstellung möglichst umfassender maschinenlesbarer Bestandsnachweise nun die verzeichneten Objekte selbst zu digitalisieren, wurde um das Jahr 2000 zum nächsten zentralen Entwicklungsziel der deutschen Bibliotheken (Altenhöner u. a. 2014). Zuerst innerhalb ihrer Abteilung „Deutsche Fotothek“ baute auch die SLUB planvoll ein leistungsstarkes Digitalisierungszentrum auf (Bove 2004).

Scanroboter und Auflichtscanner für die massenhafte Digitalisierung von Drucken sowie vielfältige Spezialgeräte für besondere Informationsträger und Formate oder besonders sensible Bestände gewährleisten heute, dass nahezu sämtliche Medientypen im Haus hoch professionell bearbeitet werden können. Das Dresdner Digitalisierungszentrum dient dem Bestandsaufbau der SLUB, sollte aber von Beginn an auch speziell für kleinere sächsische Einrichtungen wertvolles Kulturgut bearbeiten sowie als Kompetenz- und Beratungszentrum für alle Fragen der Digitalisierung fungieren. In begrenztem Umfang hatte die SLUB daher bereits vor dem Start eines offiziellen Landesdigitalisierungsprogramms entsprechende Objekte aus Chemnitz, Görlitz oder Glashütte berücksichtigt und mit Provenienzvermerken jeweils innerhalb ihrer Digitalen Sammlungen präsentiert.

Im Interesse einer möglichst selbsttragenden Entwicklung entschied sich die Bibliothek beim Aufbau ihres Digitalisierungsworkflows bewusst gegen den anderenorts öfter bevorzugten, „in jeder Hinsicht bequemeren und sichereren Einstieg“ (Syré 2012, S.179) mit einer kommerziellen Lösung, sondern investierte von Beginn an weniger in Sachleistungen an externe Wissensträger als in den internen technologischen Knowhow-Aufbau. Dabei war auch die Überzeugung leitend, dass angesichts der vielfältigen Herausforderungen der digitalen Informationsinfrastruktur und des damit verbundenen Aufgabenwandels ein entschlossener Umbau des tradierten Personalkörpers und die Heranbildung von Datenformat-, Webdesign- und Content Management-Spezialisten ohnehin unausweichlich sein würden (Bonte 2014). Zur Workflow-Unterstützung und Präsentation der Digitalisate setzt die SLUB seit 2007 deshalb die Softwaresuite „Goobi“ ein, die sie bis heute mit anderen Anwendern kooperativ weiterentwickelt. Goobi ist quelloffen, plattformunabhängig und lizenzkostenfrei und ermöglicht Digitalisierungsprojekte in großen wie kleinen Bibliotheken, Archiven, Museen und Dokumentationszentren. Die Software ist flexibel für sehr unterschiedliche 
Digitalisierungsstrategien und skalierbare Geschäftsmodelle geeignet und wird gegenwärtig bereits in rund fünfzig europäischen Wissenschafts- und Kultureinrichtungen benutzt (Bonte und Kluge 2008; Bonte 2012). Mit der Gründung eines gleichnamigen gemeinnützigen Vereins erhielt die Anwendergemeinschaft von Goobi im September 2012 eine verlässliche Struktur und ein nachhaltiges Release Management. Im Sommer 2015 umfassten die Digitalen Sammlungen der SLUB über 100.000 digitalisierte Handschriften, Notenautografe und Drucke, 20.000 Tonaufnahmen sowie rund 1,6 Millionen grafische Objekte (Fotos, Karten, Zeichnungen).

Nach der Bayerischen Staatsbibliothek ist die SLUB unter den deutschen Kultur- und Wissenschaftseinrichtungen damit der zweitgrößte Content Provider für die Deutsche Digitale Bibliothek, die Europeana und andere Aggregatoren. Die medientypologische Vielfalt der präsentierten Objekte ist dank der frühzeitigen Bearbeitung von grafischen Medien und Tonträgern deutschlandweit unübertroffen. Verfügbare digitale Kollektionen wie die „Meilenblätter von Sachsen 1780-1806“, die „Sächsischen Landtagsprotokolle 1831-1952“, die historischen Adressbücher und Stadtpläne Sachsens oder das „Archiv der Stimmen“ der Königlichen Hofoper bilden auch unter inhaltlichem Aspekt eine hervorragende Startmenge für das sächsische Digitalisierungsprogramm und zeigen im Übrigen, dass der gelegentlich konstruierte Gegensatz zwischen „Massendigitalisierung“ und „Qualitätsdigitalisierung“ (Dreis 2012, S. 89) de facto keineswegs bestehen muss.

Mit ihrem besonders wertvollen Altbestand engagierte sich die Universitätsbibliothek Leipzig bislang bevorzugt auf dem Gebiet der Digitalisierung von Handschriften und frühen Drucken. $\mathrm{Zu}$ nennen sind etwa das internationale Kooperationsprojekt „Codex Sinaiticus“, die Digitalisierung der Leipziger Papyrussammlung oder mehrere Projekte der Bibliothek zur Digitalisierung von mittelalterlichen Handschriften im Rahmen ihrer Funktion als eines von sechs DFGgeförderten deutschen Handschriftenzentren. Die Leipziger entschieden Anfang 2015, künftig ebenfalls mit Goobi zu arbeiten, was die enge Zusammenarbeit zwischen den beiden größten sächsischen Bibliotheken künftig noch leichter macht. Die Universitätsbibliothek in Freiberg hatte sich bereits 2012 zum selben Schritt entschlossen und ist aktuell mit mehreren Kollektionen zu Bergbau und Hüttenwesen online, darunter zum Beispiel 220 historische Risse sächsischer Grubenanlagen (Löwe 2014).

Die Geschichte zuverlässiger Langzeitarchive für digitale Objekte ist noch ein wenig jünger als die der systematischen Retrodigitalisierung. In einigen Regionen scheint sie gar kaum begonnen zu haben. Während eine professionelle redundante Speicherung digitaler Objekte im aktuellen Format (Bitstream Preservation) wohl meist erreicht ist, fehlen weithin Verfahren, die die Interpretierbarkeit 
der Inhalte über längere Zeiträume sicherstellen (Content Preservation). Digitale Langzeitarchivierung im strengen Sinn umfasst indes neben technischen Maßnahmen zum Erhalt der Korrektheit auch bibliothekarische und technische Maßnahmen zur nach haltigen Interpretierbarkeit digitaler Dokumente. Auf der Grundlage internationaler Forschungsprojekte, Prototypen und Standards wurden in den letzten Jahren erste entsprechende Langzeitarchive etabliert. Produktiv sind inzwischen zum Beispiel die Deutsche Nationalbibliothek, die Bayerische Staatsbibliothek und - seit Ende 2014 - auch die SLUB (Bonte und Berthold 2013). Die retrodigitalen Sammlungen und der Publikationsserver der SLUB umfassen derzeit ein Datenvolumen von rund 200 TB. Nach gründlicher Prüfung der verfügbaren Optionen hat die Bibliothek die Langzeitarchivierungslösung Rosetta der Firma Ex Libris eingeführt. Während sie selbst für Software und Workflowmodellierung verantwortlich zeichnet, wird die IT-Infrastruktur für das Produktivsystem vom Zentrum für Informationsdienste und Hochleistungsrechnen (ZIH) der TU Dresden betrieben. Im Zuge des Landesdigitalisierungsprogramms erweiterte die SLUB 2015 ihre lokale Rosetta-Lizenz auf eine Landeslizenz. Da der Betrieb eines echten Langzeitarchivs personell wie sächlich höchst aufwändig ist und wohl auch mittel- und langfristig nur von wenigen Leistungszentren gemeistert werden kann, will die SLUB im Rahmen von Absprachen mit anderen öffentlichrechtlichen Anbietern künftig auch überregional Langzeitarchivierungsdienste anbieten. Angestrebt wird, innerhalb eines Leistungsverbundes Langzeitverfügbarkeit ein abgestimmtes Angebot von passgenauen Dienstepaketen für verschiedene Systeme, Sparten und Fächer zu erbringen - von der Beratung, über die Validierung digitaler Dokumente oder der Einrichtung einer manuellen Übergabe bis hin zur Implementierung einer automatisierten Archivierungslösung. Hinsichtlich der Goobi-Anwendergemeinschaft ist das Ziel bereits greifbar nahe: Das professionelle Zusammenspiel der beteiligten Komponenten Goobi.Production und Goobi.Presentation sowie der Goobi-Workflow im SLUB-Langzeitarchiv wurden erst kürzlich erfolgreich mit dem Data Seal of Approval ausgezeichnet, einem auf internationalen Vereinbarungen beruhenden, grundlegenden Zertifikat im Bereich der digitalen Langzeitarchivierung.

\section{Eckpunkte des sächsischen Landesdigitalisierungsprogramms}

Auch in Sachsen resultierten die Ergebnisfortschritte bis 2015 überwiegend aus erfolgreichen Drittmitteleinwerbungen, namentlich von der Deutschen Forschungsgemeinschaft (Bürger 2011; Bürger 2013), hinsichtlich der technologi- 
schen Verbesserung der Informationsinfrastruktur zudem aus EFRE-Mitteln. Angesichts von Produktionskosten zwischen ca. 50 Euro und 150 Euro pro Band, je nach Umfang und Erschließungstiefe, sowie einem zu Anfang des Jahrzehnts geschätzten gesamtdeutschen Investitionsbedarf von über 30 Millionen Euro pro Jahr (Gesamtkonzept Informationsinfrastruktur 2011, S. 38) war jedoch früh klar, dass diese Fördermittel kraftvoll flankiert werden müssten, wenn die Transformation der gedruckten Überlieferung in vertretbarer Zeit realisiert werden sollte. Unter Verweis auf die Bedeutung der Aufgabe, das große Engagement der DFG und die notwendige Festigung der überregional beachteten Aufbauerfolge konnte der Generaldirektor der SLUB das Sächsische Staatsministerium für Wissenschaft und Kunst (SMWK) im Frühjahr 2014 für eine planungssichere Mitfinanzierung aus Landesmitteln gewinnen. Mit der Verabschiedung des Landeshaushalts 2015/16 sowie einer zwischen dem SMWK und der SLUB abgestimmten Verfahrensrichtlinie wird das sogenannte „Landesdigitalisierungsprogramm für Wissenschaft und Kultur des Freistaates Sachsen“ seit Mai 2015 operativ vorangetrieben. Die Fortsetzung des Programms in den Haushaltsjahren 2017/18 ff. ist vorgesehen.

Da es in ganz Deutschland bislang kaum gelungen ist, „,nachhaltige und vor allem langfristig finanzierte Programme zur Digitalisierung von Kulturgut in Bibliotheken, Archiven und Museen ins Leben zu rufen“ (Altenhöner u. a. 2014, S. 775), ist Sachsen dank dieser politischen Entscheidung eines der ersten Bundesländer mit einer verbindlichen Unterstützung und Koordination von Digitalisierungsprojekten. Mit der „Servicestelle Digitalisierung“ am Konrad-Zuse-Zentrum für Informationstechnik hatte 2012 schon Berlin diesen Weg eingeschlagen. Während sich die Berliner Initiative durch einen ausdrücklich spartenübergreifenden Ansatz auszeichnet und in einem antragsbasierten jährlichen Förderprogramm mit Fachjury vollzieht, erprobt Sachsen bis auf Weiteres einen sehr pragmatischen Weg und weist der Staatsbibliothek auch stärker projektstimulierende, inhaltlich auswählende und andere aktiv gestaltende Funktionen zu. In konsequenter Verfolgung der Grundidee für das wissenschaftliche Bibliothekswesen (Bonte und Linek 2008) wird obendrein der strukturbildende Aspekt beachtet, der darauf zielt, landesweit künftig möglichst einheitliche Werkzeuge zur Produktion, Präsentation, Langzeitarchivierung und Weiterverarbeitung (Volltexterkennung, Volltextindexierung, Social Tagging u.a.) von Digitalisaten einsetzen zu können.

Mit zusätzlichen Haushaltsmitteln in Höhe von jeweils 2,5 Millionen Euro wird der Freistaat 2015 und 2016 in drei Aktionslinien den Wandel zur digitalen Wissensgesellschaft vorantreiben. Erstens soll die kooperative Lizenzierung digitaler Medien gestärkt (Erwerbungskonsortium der sächsischen Hochschulbibliotheken), zweitens die retrospektive Digitalisierung von bedeutenden analo- 
gen Medien ausgebaut, drittens die Langzeitverfügbarkeit der digitalen Objekte gesichert werden. Erklärtes Ziel der Landesregierung ist, mit der signifikanten Steigerung von frei zugänglichen digitalen Volltexten die Bekanntheit des sächsischen Kulturerbes zu mehren, Wissenschaft, Forschung und kulturelle Bildung zu unterstützen sowie die Kooperations- und Drittmittelfähigkeit der sächsischen Kultur- und Wissenschaftseinrichtungen weiter zu verbessern.

Im Interesse maximalen Servicenutzens sollen im Bereich der Retrodigitalisierung nicht nur gemeinfreie, sondern auch urheberrechtlich geschützte Objekte bearbeitet werden können. Aus demselben Grund können prinzipiell auch Einrichtungen profitieren, die nicht in Landesträgerschaft stehen. Eine Digitalisierung ist zudem nicht nur für Drucke und Handschriften möglich, sondern zum Beispiel auch für audiovisuelle Medien wie etwa Zelluloidfilme, Glas- oder Schellackplatten. In jedem Fall werden ausreichende Metadaten und hinreichende Digitalisierungsfähigkeit aus konservatorischer Sicht vorausgesetzt. Für die technischen Standards der Projekte gelten die DFG-Praxisregeln „Digitalisierung“ in ihrer jeweils aktuellen Fassung. Neben der Einbindung der Digitalisate in eigene Oberflächen willigt die besitzende Institution in die integrierte Präsentation der Objekte aus dem Landesdigitalisierungsprogramm sowie in die Bereitstellung für die Deutsche Digitale Bibliothek (DDB), Europeana und vergleichbare Aggregatoren ein. Die digitalen Werke werden - soweit möglich - unter einer offenen Lizenz publiziert. Mit der Koordination des Landesdigitalisierungsprogramms ist die SLUB beauftragt, die die Mittel verwaltet und in Absprache mit dem SMWK sowie in engem Kontakt mit Fachgremien wie der Konferenz der sächsischen Bibliotheksdirektorinnen und -direktoren auch deren bedarfsgerechte Verteilung auf die drei Aktionslinien steuert. Die Koordinierungs- und Servicefunktionen entsprechen dem gesetzlichen Auftrag der SLUB und fußen auf den langjährigen Erfahrungen in allen drei Arbeitsbereichen. Die Bibliothek verfügt über skalierbare Digitalisierungs- und Langzeitarchivierungsworkflows, leitet das Erwerbungskonsortium der sächsischen Hochschulbibliotheken und kann mit ihrer bereits 1996 eingerichteten „Landesstelle für Bestandserhaltung“ auch die Beachtung konservatorischer Aspekte sowie im Einzelfall notwendige flankierende Maßnahmen zum Originalerhalt wertvoller Objekte gewährleisten (Schäfer und Vogel 2014).

Die Digitalisierung der ausgewählten Objekte wird bevorzugt von Dienstleistern auf der Basis von Goobi durchgeführt. Ausnahmen gelten zum Beispiel für die wertvollen Handschriftenbestände in Leipzig, da die Universitätsbibliothek selbst über das notwendige Spezialgerät verfügt, oder für besonders schwierige Materialien, die in der SLUB digitalisiert werden. Unter den Dienstleistern sind besonders Generalunternehmer gefragt, die neben dem eigentlichen Scanprozess jeweils vor Ort auch an der Projektvorbereitung und Goobi-Workflowmodel- 
lierung mitwirken. Die Belastbarkeit dieser Methode wird gegenwärtig im Wege von Entwicklungspartnerschaften erprobt und soll anschließend als Rahmenvereinbarung offen ausgeschrieben werden.

\section{Erste Maßnahmen}

Bei der Umsetzung des Programms stützt sich die SLUB auf bewährte Hilfsmittel und Strukturen. In Erweiterung der hausinternen Koordinierungsstelle für die Digitale Bibliothek wurde eine Geschäftsstelle eingerichtet, die interessierte Einrichtungen beraten, die Erarbeitung der konkreten Projekte begleiten und bei der Auftragsvergabe und Workflowmodellierung mitwirken kann. Die Geschäftsstelle hat erste informierende Webseiten publiziert sowie eine Checkliste entwickelt, mit der Interessierte die grundsätzliche Eignung des gewünschten Objekts bzw. ihrer Sammlung für das Digitalisierungsprogramm selbst einschätzen können. Auf den Seiten werden künftig auch sämtliche realisierten Projekte aufgelistet und beschrieben. Weitere Vorbereitungen betrafen die Lizenzerweiterung für die Langzeitarchivierungslösung Rosetta für ganz Sachsen, Hardwareinvestitionen im Dresdner Digitalisierungszentrum sowie die Erarbeitung eines Personalkonzepts für die in der SLUB zu beteiligenden Expertenteams (Goobi-Workflow, Strukturmetadaten/OCR u.a.).

Während die Möglichkeiten zur Massendigitalisierung von Drucken im Bereich der gemeinfreien Werke für die SLUB bereits weitgehend ausgeschöpft sind, bieten vor allem die Bestände der Universitätsbibliothek Leipzig auf diesem Gebiet noch interessante Chancen. Kurz nach Programmstart richten sich die inhaltlichen Vorbereitungen zur Retrodigitalisierung daneben zum Beispiel auf die extrem gefährdeten Filme des Tanzarchivs Leipzig. Ferner werden die historischen Landtagsakten geprüft, die die bereits digitalisierten Landtagsprotokolle 1831-1933 ergänzen sollen, sowie einige große sächsische Zeitungen, die aus dem Mikrofilmarchiv der SLUB digitalisiert werden können. Darüber hinaus sind bedeutende Musikhandschriften und -drucke angefragt, die sich zum Teil bereits als Depositalbestand in der SLUB befinden, schließlich der Lusatica-Bestand der Oberlausitzischen Akademie der Wissenschaften in Görlitz sowie Materialien der Fachhochschulbibliothek Zwickau zur Geschichte der Textilindustrie. Inwiefern sich alle genannten Projekte noch 2015 zumindest anschieben lassen werden, wird die nähere Zukunft zeigen. Ziel ist freilich, schon Ende des Jahres erste Referenzprojekte zu haben, um den hohen Wert des Landesdigitalisierungsprogramms rasch zu veranschaulichen. 


\section{Perspektiven}

Das sächsische Landesdigitalisierungsprogramm wird in Wissenschaft und Öffentlichkeit wirken, verspricht aber auch für die Gedächtniseinrichtungen selbst drei wesentliche, nachhaltige Erträge: Erstens können der Anteil digital verfügbarer Objekte rascher vermehrt, zweitens die Standardisierung von Datenformaten und Geschäftsprozessen im Land gefestigt bzw. ausgebaut und drittens Interesse und Verständnis für Aufgaben der digitalen Informationsinfrastruktur angeregt werden. Darüber hinaus sind zusätzliche Impulse für verbesserte Verfahren zur Informationsrecherche und -visualisierung vorstellbar. Im Zuge der breiten Bemühungen, aus den Reproduktionen von Texten nicht dauerhaft nur Bilder, sondern leicht prozessierbare Texte herzustellen (Federbusch und Polzin 2013), sollen die Digitalisate so weit wie möglich als Volltexte indexiert bzw. recherchiert werden können. Die SLUB begleitet entsprechend engagiert die Förderinitiative der DFG zur Weiterentwicklung von Verfahren für die Optical-Character-Recognition (OCR) und wird noch 2015 ihr Präsentationsmodell in Goobi voll für die wahlweise Anzeige von Bild- wie Textdateien ausgebaut haben. Die Recherche der Volltexte wird durch laufende Projekte zur quelloffenen Entwicklung von Datenmanagement-Werkzeugen und Discovery-Software unterstützt, die die SLUB und die Universitätsbibliothek Leipzig mit anderen, dezidiert an offenen Systemen interessierten Einrichtungen vorantreiben. Im Wege der Datenintegrations- und modellierungslösung „d:swarm“ und bereits publizierter, nicht kommerzieller Discovery-Oberflächen (VUFind - „finc“ (Leipzig) bzw. TYPO3 „SLUB-Katalog“) sollen Daten aus unterschiedlichsten Quellsystemen auf einfache Weise und in hoher Qualität miteinander verknüpft und angereichert werden. Ziel ist die Herstellung eines spezifischen Wissensgraphen, der Kataloge und Präsentationssysteme versorgt und zugleich als Linked Open Data zur Nachnutzung bereitsteht (Lohmeier 2015).

Das Digitalisierungsprogramm bietet schließlich auch Anreize für den Ausbau von Citizen Science-Konzepten, die speziell für Landesbibliotheken und Wissenschaftliche Stadtbibliotheken neue Horizonte öffnen könnten. Als Citizen Science oder Bürgerwissenschaft werden wissenschaftsrelevante Projekte bezeichnet, die unter Mitwirkung von interessierten Laien vorankommen - sei es durch Sammeln oder Anreichern von Daten (Crowdsourcing), sei es durch qualifizierte Formulierung von Forschungsfragen und Erarbeitung von Methoden (Finke 2014). Beliebte Arbeitsfelder von Citizen Science sind etwa Genealogie und Heimatkunde oder Naturschutz und Denkmalpflege: sämtlich Bereiche, für die das Landesdigitalisierungsprogramm ohne Zweifel neue Materialien verfügbar machen wird. Welche konkreten Herausforderungen aus einzelnen Projekten der Entwicklung von Digitalisierungs- und Präsentationssoftware, Recherchefunkti- 
onen oder Citizen Science-Konzepten im Detail zusätzlichen Schwung verleihen werden, bleibt abzuwarten. Gewiss ist freilich schon heute, dass das neue Programm auch unter dieser Perspektive die historische Chance für einen weiteren, kräftigen Entwicklungsschub für die sächsische Informationsinfrastruktur bietet.

\section{Bibliographie}

Altenhöner, Reinhard u. a. 2014. Digitalisierung von Kulturgut. Praxishandbuch Bibliotheksmanagement, hg. v. Rolf Griebel u. a., 775-823. Berlin: De Gruyter Saur.

Bonte, Achim und Andreas Kluge. 2008. Massenhaft Mehrwert. Digitalisierung von Kulturgut in den sächsischen wissenschaftlichen Bibliotheken. BIS. Das Magazin der Bibliotheken in Sachsen 1 (1) 40-43.

Bonte, Achim und Joachim Linek. 2008. Bibliothekssystem Sachsen. Struktur- und Entwicklungsplan für die wissenschaftliche Literatur- und Informationsversorgung im Freistaat Sachsen. Dresden: Sächsisches Staatsministerium für Wissenschaft und Kunst.

Bonte, Achim. 2012. Fünf Jahre Goobi. Das Rückgrat der Massendigitalisierung in der SLUB. BIS. Das Magazin der Bibliotheken in Sachsen 5 (2): $84 \mathrm{f}$.

Bonte, Achim und Henrike Berthold. 2013. Zugriff für die Ewigkeit. Digitale Langzeitarchivierung an der SLUB. BIS. Das Magazin der Bibliotheken in Sachsen 6 (2) 82-84.

Bonte, Achim. 2014. Wissenschaftliche Bibliotheken der nächsten Generation. Sind die Institutionen und ihre Mitarbeiter für die Zukunft gerüstet? Zeitschrift für Bibliothekswesen und Bibliographie 61 (4/5) 239-242.

Bove, Jens. 2004. Fotothek digital. SLUB-Kurier 18 (2) $7 \mathrm{f}$.

Bürger, Thomas. 2011. Die Digitalisierung der kulturellen und wissenschaftlichen Überlieferung - Versuch einer Zwischenbilanz. Zeitschrift für Bibliothekswesen und Bibliographie 58 (3/4) 133-141.

Bürger, Thomas. 2013. Die Digitale Transformation weiter gestalten. Der Beitrag der DFG zu einer innovativen Informationsinfrastruktur für die Forschung. Bibliotheken - Tore zur Welt des Wissens. Kongressband zum 101. Dt. Bibliothekartag in Hamburg 2012, hg. v. Klaus-Reiner Brintzinger, 19-29. Hildesheim: Olms

Dreis, Gabriele. 2012. Wissen mehren und verbreiten: Zur Digitalisierung schriftlichen Kulturguts in der Universitäts- und Landesbibliothek Düsseldorf. Digitalisierung in Regionalbibliotheken, hg. v. Irmgard Siebert, 87-115. Frankfurt/M.: Klostermann

Federbusch, Maria und Christian Polzin. 2013. Volltext via OCR. Möglichkeiten und Grenzen. Berlin: Staatsbibliothek zu Berlin - Preußischer Kulturbesitz Hg.

Finke, Peter. 2014. Citizen Science: Das unterschätzte Wissen der Laien. München: Oekom Gemeinsame Wissenschaftskonferenz des Bundes und der Länder. 2011. Gesamtkonzept für die Informationsinfrastruktur in Deutschland. Empfehlungen der Kommission Zukunft der Informationsinfrastruktur im Auftrag der Gemeinsamen Wissenschaftskonferenz http://www.leibnizgemeinschaft.de/fileadmin/user_upload/downloads/Infrastruktur/ KII_Gesamtkonzept.pdf.

Karlsch, Rainer und Michael Schäfer. 2006. Wirtschaftsgeschichte Sachsens im Industriezeitalter. Leipzig: Edition Leipzig. 
Löwe, Oliver. 2014. Sächsisches Berg- und Hüttenwesen digital. Universitätsbibliothek Freiberg startet Digitale Bibliothek mit Goobi. BIS. Das Magazin der Bibliotheken in Sachsen 7 (2) $108 \mathrm{f}$.

Lohmeier, Felix. 2015. Datenintegration mit D:SWARM. Werkstattbericht zum Management bibliothekarischer Daten an der SLUB Dresden. BIS. Das Magazin der Bibliotheken in Sachsen 8 (2) $88 \mathrm{f}$.

Schäfer, Irmhild und Michael Vogel. 2014. Restaurierung und Bestandserhaltung. Praxishandbuch Bibliotheksmanagement, hg. v. Rolf Griebel u. a., 837-861. Berlin: De Gruyter Saur.

Schneider, Ulrich Johannes. 2009. Ein Kosmos des Wissens. Weltschrifterbe in Leipzig. Leipzig: Universitätsbibliothek.

Siebert, Irmgard. 2012. Hg: Digitalisierung in Regionalbibliotheken. Frankfurt/M.: Klostermann. Syré, Ludger. 2012. Aufbruch in eine neue Zeit: Die Anfänge der Digitalisierungswerkstatt und der „Digitalen Sammlungen“ an der Badischen Landesbibliothek in Karlsruhe. Digitalisierung in Regionalbibliotheken, hg. v. Irmgard Siebert, 173-194. Frankfurt/M.: Klostermann.

Winterfeld, Klaus. 2006. Das sächsische Kulturraumgesetz. Eine Bilanz nach elf Jahren. Leipzig: Leipziger Universitätsverlag.

\section{Abbildungen}

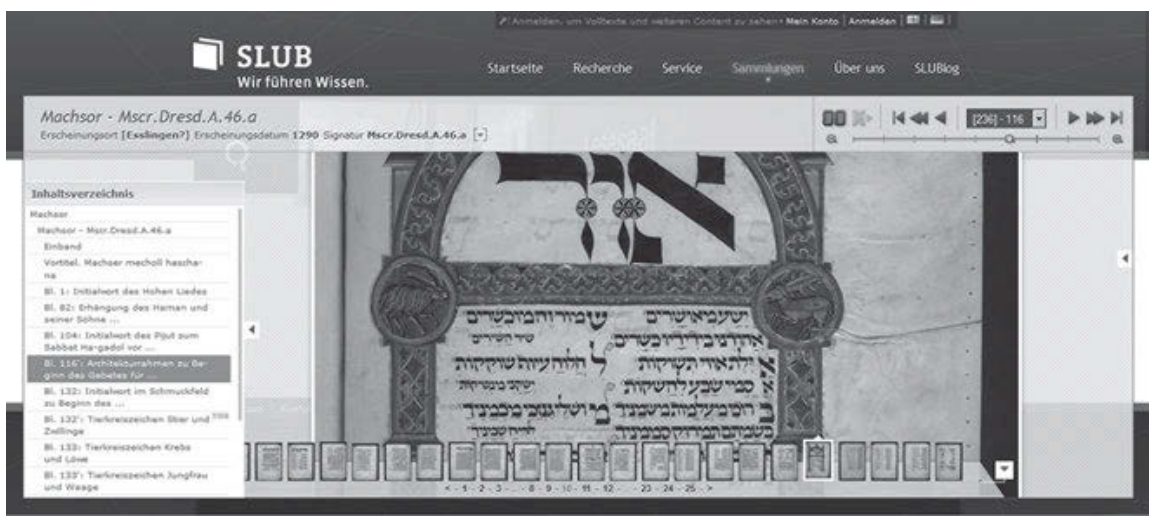

Abb. 1: Digitale Sammlungen. 


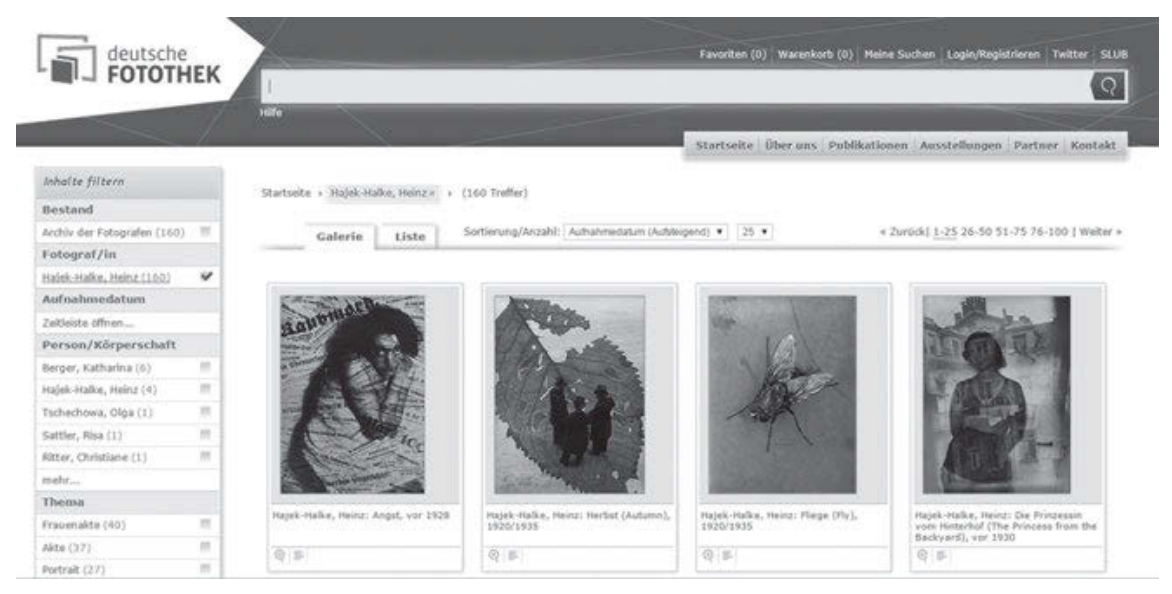

Abb. 2: Deutsche Fotothek.

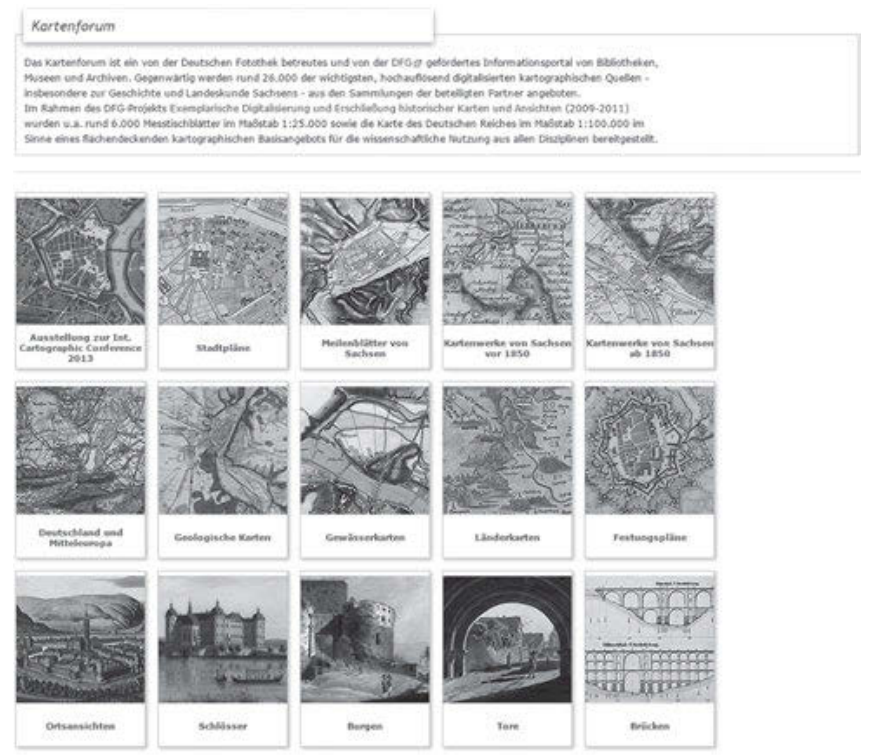

Abb. 3: Kartenforum. 


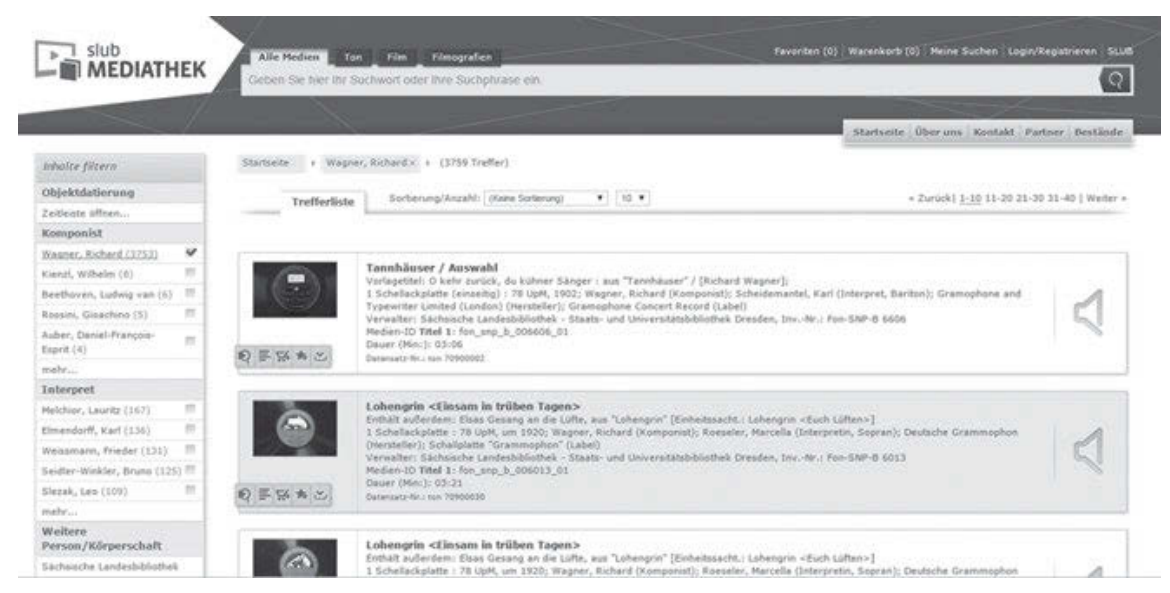

Abb. 4: Archiv der Stimmen. 